\title{
Improvement in Patient Mental Well-being After Surgery for Cervical Spondylotic Myelopathy
}

Tamai Koji, Suzuki Akinobu, Terai Hidetomi, Hoshino Masatoshi, Toyoda Hiromitsu, Takahashi Shinji, Ohyama Shoichiro, Hori Yusuke, Yabu Akito, Nakamura Hiroaki

\begin{tabular}{|c|c|}
\hline Citation & SPINE. 45(10); E568-E575 \\
\hline Issue Date & $2020-05-15$ \\
\hline Type & Journal Article \\
\hline Textversion & Author \\
\hline Key Points & $\begin{array}{l}\text { The mental well-being of patients with CSM is impaired, and, on average, showed } \\
\text { meaningful improvement after laminoplasty. } \\
\text { - The trend toward improvement was sustained for } 2 \text { years, but not for } 5 \text { years. } \\
\text { - In terms of individual cases, half of the patients achieved meaningful } \\
\text { improvement. } \\
\text { - A lower preoperative "SF" score, based on a cut-off value of 50, was an } \\
\text { independent predictor of significant improvement in mental well-being. } \\
\text { - These results suggest that cervical myelopathy may have the greatest } \\
\text { detrimental effect on social function among the numerous aspects of mental } \\
\text { well-being. }\end{array}$ \\
\hline Relation & $\begin{array}{l}\text { This is the peer-reviewed but unedited manuscript version of the following article: } \\
\text { SPINE: 2020. Vol.45, Issu.10, E568-E575. The final, published version is available at } \\
\text { https://doi.org/10.1097/BRS.0000000000003337. }\end{array}$ \\
\hline DOI & 10.1097/BRS.0000000000003337 \\
\hline
\end{tabular}

\section{Self-Archiving by Author(s)}

\section{Placed on: Osaka City University Repository}

Tamai, K., Suzuki, A., Terai, H., Hoshino, M., Toyoda, H., Takahashi, S., Ohyama, S., Hori, Y., Yabu, A., \& Nakamura, H. (2019). Improvement in Patient Mental Well-being After Surgery for Cervical Spondylotic Myelopathy. Spine, 45(10), E568-E575. https://doi.org/10.1097/brs.0000000000003337 
Improvement in patient mental well-being after surgery for cervical spondylotic myelopathy

Koji Tamai MD, Akinobu Suzuki MD, Hidetomi Terai MD, Masatoshi Hoshino MD, Hiromitsu Toyoda

MD, Shinji Takahashi MD, Shoichiro Ohyama MD, Yusuke Hori MD, Akito Yabu MD, Hiroaki

Nakamura MD

Department of Orthopedics, Osaka City University Graduate School of Medicine, Osaka, Japan

Corresponding author:

Koji Tamai MD

Department of Orthopedics, Osaka City University Graduate School of Medicine, Osaka, Japan

Phone: +81-6-6645-3851, Email: koji.tamai@msic.med.osaka-cu.ac.jp

The submitted manuscript does not contain any information about medical drugs.

Conflict of Interest and Source of Funding: No funds were received in support of this work.

No benefits in any form have been or will be received from a commercial party related directly or

indirectly to the subject of this manuscript.

The study protocol was approved by the Institutional Review Board of Osaka City University. 


\section{Abstract (299/300)}

\section{Study Design}

Retrospective cohort study

\section{Objective}

To investigate changes in mental well-being after surgery for cervical spondylotic myelopathy (CSM) and identify factors associated with improvement.

\section{Summary of Background Data}

Posterior cervical surgery with laminoplasty significantly improves myelopathy and physical function in patients with CSM. However, its impact on mental well-being is unclear.

\section{Methods}

Patients who underwent laminoplasty for CSM and had >2 years of follow-up were reviewed (n=111).

The mental component summary (MCS) score was used as a measure of mental well-being. The trend in MCS score change was evaluated using the Jonckheere-Terpstra trend test. Preoperative clinical scores were compared between patients with improvements greater and less than the minimal clinically important difference (MCID). Significant variables were included in a multinomial logistic regression analysis and further validated in a receiver-operating characteristic (ROC) curve analysis. Additionally, the results were confirmed in a long-term observation cohort of patients followed up for $>5$ years $(n=46)$.

\section{Results}


The improvement in the average MCS score (5.6) was greater than the MCID (4.0). The trend of improvement was sustained for 2 years $(\mathrm{p}=0.002)$, but not for 5 years $(\mathrm{p}=0.130)$. In terms of individual cases, 56 patients $(50.5 \%)$ achieved MCS score improvement greater than the MCID. These patients showed significantly lower preoperative MCS scores than those without meaningful improvement $(\mathrm{p}<0.001)$. The preoperative "social functioning $(\mathrm{SF})$ " score was independently associated with MCS score improvement $(\mathrm{p}=0.001)$. ROC curve analysis validated the ability of preoperative SF to predict MCS score improvement at 2 and 5 years postoperatively (area under the curve: 0.744, 0.893, respectively).

\section{Conclusion}

Half of the patients achieved meaningful improvement in mental well-being. A lower preoperative SF score was independently associated with improvement. These results may help identify patients who could experience an improvement in mental well-being after surgery and develop novel approaches to achieve further improvement.

Level of evidence: III (Prognosis: Cohort study)

\section{Keywords:}

laminoplasty; cervical spondylotic myelopathy; mental health; mental well-being; social functioning; 
minimum clinical important differences; posterior decompression,

\section{Key points}

- The mental well-being of patients with cervical spondylotic myelopathy is impaired, and, on average, showed meaningful improvement after laminoplasty.

- The trend towards improvement was sustained for 2 years, but not for 5 years.

- In terms of individual cases, half of the patients achieved meaningful improvement.

- A lower preoperative "social functioning" score, based on a cut-off value of 50, was an independent predictor of significant improvement in mental well-being.

- These results suggest that cervical myelopathy may have the greatest detrimental effect on social function among the numerous aspects of mental well-being.

\section{Mini summary}

The mental well-being of patients with cervical spondylotic myelopathy showed, on average, meaningful improvement after laminoplasty, which was sustained for 2 years. In terms of individual cases, half of the patients achieved meaningful improvement after laminoplasty. A lower preoperative "social functioning" score was independently associated with improvement. 


\section{Introduction}

Cervical spondylotic myelopathy (CSM) is a degenerative spinal disorder that compresses the spinal

cord and leads to the deterioration of neurological function. Surgical decompression is the gold standard

procedure for preventing the progression of neurological deficits in patients with $\mathrm{CSM}^{1}$. Among several

surgical techniques, laminoplasty is generally recognized as the most effective surgical option, with the

lowest risk of perioperative complications ${ }^{2-6}$. The current treatment trends in the United States show that

laminoplasty is the third most commonly performed procedure ${ }^{1}$.

The aim of surgery for patients with CSM should be to improve myelopathy or prevent further deterioration of the myelopathy and neurological symptoms. However, surgical satisfaction can sometimes be independent from the quality and effectiveness of the surgery, as it is related to numerous factors, including age, sex, education, lifestyle, expectations, psychological distress, and individual values $^{7-10}$. Among these factors, mental health status or mental well-being has been reported as one of the most important for patient satisfaction with overall care ${ }^{11}$. Patients with psychological distress report significantly lower scores for overall satisfaction and satisfaction with their provider than do those without psychological distress ${ }^{11}$.

Therefore, understanding the mid- and long-term changes in mental well-being after cervical laminoplasty 
would be useful to physicians. Furthermore, identifying the preoperative predictors of improvement in mental well-being can help surgeons to predict mental well-being changes, develop targeted postoperative interventions, and provide better explanations of the changes that patients would experience after surgery, which can result in higher patient satisfaction ${ }^{12}$. Therefore, the aim of this study was to investigate the change in mental well-being at 2 and 5 years after laminoplasty. Additionally, we set out to determine the preoperative factors associated with an improvement in mental well-being. 


\section{Methods}

We performed a retrospective cohort study of patients who underwent laminoplasty for CSM. All study participants provided informed consent, and the study protocol was approved by the Institutional Review Board at our institution (No. 3170).

Main-group population (2-year cohort)

We reviewed 132 patients who underwent C3-C6 laminoplasty for CSM at our institution between 2008 and 2012, and were followed up for more than 2 years postoperatively. Patients were excluded if any of their preoperative or 2-year follow-up data were missing $(n=16)$ or there was an obvious history of mental disease, such as depression $(n=4)$ or schizophrenia $(n=1)$, in their medical record. A total of 111 patients were included in the final analysis (65 women, 46 men; mean age at surgery, $65.2 \pm 11.7$ years).

\section{Sub-group population (5-year cohort)}

Among the 111 patients in the 2-year cohort, those with available preoperative 6-month, 1-year, 2-year, as well as 5-year clinical follow-up data were further analyzed as part of the 5-year cohort. A total 46 patients were included in the 5-year cohort (30 women, 16 men; mean age at surgery, 64.2 \pm 9.6 years).

\section{Surgical procedure}


All patients underwent open door laminoplasty ${ }^{13}$. The surgical indication and approach were decided upon on a case-by-case basis by the treating physicians. Patients with severe dementia (Hasegawa's Dementia Scale-Revised score $<20$ points $^{14}$ ) were not indicated for surgical therapy. Hydroxyapatite spacers or anchor screws were used at each level to fix the opened lamina ${ }^{15}$. The day after surgery, all patients were allowed to sit up with a soft neck collar and to stand and walk. Removal of the soft brace was allowed 1 week after surgery. All patients were then encouraged to start range of motion and isometric muscle strengthening exercises of the neck as early as possible.

\section{Clinical evaluation}

The cervical Japanese Orthopaedic Association (cJOA) score, which is a physician-assessed scoring system of the severity of myelopathy, was evaluated preoperatively and at 6 months, 1 year, and 2 years postoperatively, and at 5 years if possible ${ }^{16}$. Patient-oriented questionnaire scores, including the visual analog scale (VAS) for neck and arm pain and arm numbness, and the 36-item Short-Form Health Survey (SF-36), were performed preoperatively and at 6 months, 1 year, and 2 years postoperatively, and at 5 years if possible. The SF-36 is a 36-item scale measuring 8 domains of health-related quality of life: physical functioning $(\mathrm{PF})$, physical role limitations (RP), bodily pain (BP), general health perceptions (GH), energy/vitality (VT), social functioning (SF), emotional role limitations (RE), and mental health $(\mathrm{MH})^{17}$. The domain scores were then summarized as the physical component summary (PCS) and mental 
component summary (MCS) scores, according to an algorithm proposed by a previous report ${ }^{18}$.

\section{Mental well-being parameter}

We defined the MCS score of the SF-36 as the parameter of mental well-being in this study ${ }^{19}$. The average and standard deviation of the MCS score in healthy Japanese individuals between the ages of 60 and 65 years has been reported as $52.4 \pm 9.8^{20}$. The minimal clinically important difference (MCID) in the MCS score was defined as an improvement of more than $4.0^{21}$.

\section{Study design \& statistical analysis}

\section{Main analysis using the 2-year cohort}

The change in the average of each clinical score from preoperatively to 2 years postoperatively was evaluated using the paired t-test. The trend of MCS score improvement from preoperatively to 6 months, 1 year, and 2 years postoperatively was analyzed using the Jonckheere-Terpstra trend test. To evaluate individual cases, patients were then divided into 2 groups according to their change in the MCS score, with the MCID as the cut-off point. The no-change group included patients whose MCS score improved by less than the MCID, while the improvement group included those with an improvement of more than the MCID. The preoperative and 2-year postoperative MCS scores were compared between the two groups using the Mann-Whitney $U$ test. In addition, age, sex, and all components of the preoperative 
cJOA score (finger motion, upper extremity motion, lower extremity motion, upper extremity sensory,

trunk sensory, lower extremity sensory, bowel bladder dysfunction, and total score) and preoperative

SF-36 (PF, RP, BP, GH, VT, SF, RE, and MH) were compared between the two groups using the Mann-Whitney U test or Chi-squared test, as appropriate. Variables with a p value $<0.05$ in the univariate analysis were included in the subsequent multinomial logistic regression model as the explanatory variables. The improvement group was set as the objective variable. Subsequently, continuous significant factors in logistic regression analysis were separately analyzed using receiver operating characteristic (ROC) curves to validate the results and investigate the cutoff values to predict failure to achieve the MCID. The area under the ROC curve (AUC) and 95\% confidential intervals (CIs) were calculated. An AUC of 0.5 indicates chance performance; 0.5 to 0.6 , bad predictive ability; 0.6 to 0.7 , sufficient predictive ability; 0.7 to 0.8 , good predictive ability; and 0.8 to 1.0 , excellent predictive ability ${ }^{22}$. The cutoff value was determined by measuring the distance from the top left corner of the ROC curve. In addition, the change in the cJOA score and subdomains of the SF-36 at 2 years after surgery were evaluated using the Mann-Whitney U test.

Sub-group analysis using the 5-year cohort

The change in the average MCS score from preoperatively to 5 years postoperatively was evaluated using the paired t-test. The trend of MCS score improvement from preoperatively to 6 months, 1 year, 2 years, 
and 5 years postoperatively was analyzed using the Jonckheere-Terpstra trend test. Subsequently, to validate the results of the main analysis, the relevant variables that were identified in the main analysis were submitted to a ROC curve analysis using the 5-year cohort data. The outcome of the ROC analysis was failure to achieve the MCID. All analyses were performed using SPSS software (version 23; SPSS, Chicago, IL). A p value $<0.05$ was considered statistically significant. 


\section{Results}

Main analysis

Change in overall averages

All components of the cJOA score, VAS, and SF-36 were significantly improved at 2 years postoperatively compared to preoperative values (Table 1). The average preoperative MCS score (40.7) was less than that reported by healthy controls (52.4). The MCS score changed from 40.7 preoperatively to 46.3 at 2-years postoperatively, an improvement of 5.6, which was more than the MCID. There was a significant trend in improvement from preoperatively to 6 months, 1 year, and 2 years postoperatively (Figure 1, $\mathrm{p}=0.002$ ).

\section{Comparison between the no-change and improvement groups}

Fifty-six patients (50.5\%) were classified into the improvement group and 55 (49.5\%) into the no-change group. The preoperative MCS score was significantly lower in the improvement group than in the no-change group (33.9 vs. 47.5, p <0.001). However, the 2-year postoperative MSC score was significantly higher in the improvement group than in the no-change group (49.4 vs. 43.1, $\mathrm{p}=0.012$ ). There were no significant differences between the groups in terms of age and sex (Table 2). In the univariate comparison of preoperative scores between the groups, there were no significant differences in individual component and total cJOA scores. However, the preoperative SF-36 subdomains of VT, SF, RE, 
and $\mathrm{MH}$ were significantly higher in the no-change group than in the improvement group. In the multinomial logistic regression analysis with the preoperative VT, SF, RE, and MH included as explanatory variables, the preoperative SF was found to be an independent factor related to MCS score improvement $(\mathrm{p}=0.001)$. Changes in $\mathrm{cJOA}$ scores after the surgery were not significantly different between groups. In contrast, all subdomains of the SF-36 showed greater improvement in the improvement group than in the no-change group (Table 3).

\section{ROC curve analysis}

The AUC for the preoperative SF score's ability to predict MCS score improvement was 0.744 , which indicates good predictive ability (95\% CI 0.652 to $0.836, \mathrm{p}<0.001)$ (Figure 2 ). The optimal cutoff value of the preoperative SF score for MCS score improvement prediction was 50 (sensitivity $71.2 \%$, specificity $68.6 \%)$

Sub-group analysis

Trend of average MSC score improvement

The MCS score significantly improved from 40.9 preoperatively to 45.5 at 5 years postoperatively $(\mathrm{p}<0.001)$, with a difference of 4.6, which is more than the MCID. However, in the trend analysis, the tendency of improvement from preoperatively to 6 months, 1 year, 2 years, and 5 years postoperatively 
failed to reach significance (Figure 3, $\mathrm{p}=0.130$ ).

ROC curve analysis

Based on the results of the main analysis, we evaluated whether the preoperative SF score can predict MCS score improvement at 5 years after the surgery. The ROC curve analysis revealed an AUC for the SF score of 0.893 , indicating an excellent predictive ability (95\% CI 0.788 to $0.998, \mathrm{p}<0.001$, Figure 4 ). The optimal cutoff value for the preoperative SF score was 50 (sensitivity $90.9 \%$, specificity $75.0 \%$ ). 


\section{Discussion}

In the present study, we demonstrated that the MCS score, an indicator of mental well-being, was significantly improved, on average, after laminoplasty. The trend of improvement was sustained for 2

years, but not for 5 years. In terms of individual cases, half of the patients experienced an improvement that was greater than the MCID, while the remainder failed to achieve meaningful improvement. The patients with meaningful improvement showed significantly lower preoperative MCS scores than those in patients without meaningful improvement. A lower preoperative SF score, based on a cut-off value of 50, was independently associated with an improvement in mental well-being at both 2 and 5 years postoperatively.

A clear change in mental well-being after laminoplasty has not been concluded. In contrast to our findings, a retrospective review of 90 patients by Kato et al. found no significant change in the SF-36 MCS score after laminoplasty ${ }^{23}$. One difference between this previous study and our study is the homogeneity of the diagnosis; almost $40 \%$ of the patients in the study by Kato et al. were diagnosed with ossification of the posterior longitudinal ligament (OPLL), while all patients in the present study were diagnosed with CSM. This potentially suggests that mental well-being can improve after laminoplasty for patients with CSM, but not for patients with OPLL. 
Our results showed that only half of the patients experienced a meaningful improvement in their mental well-being after surgery. In addition, these patients exhibited significantly lower MCS scores before surgery. These findings suggest that cervical myelopathy might negatively affect the mental well-being of almost half of all patients with CSM (50.5\% in current results). However, neither the preoperative severity of myelopathy nor the improvement in myelopathy, as evaluated by cJOA scores, had an impact on MCS score improvement. This suggests that the cJOA scores cannot indicate the effect of myelopathy on mental well-being; thus, it behooves physicians to consider other indicators of patient mental well-being after surgery for CSM.

Our multivariate analysis showed that the SF domain of the SF-36 was independently associated with MCS score improvement, despite the fact that MH and RE have the greatest and second-greatest influence, respectively, on the MCS score, based on the defined formula ${ }^{24,25}$. This result was validated in the sub-cohort with more than 5 years of follow-up after surgery. The SF domain, which refers to "social functioning", is defined as the "individual's interactions with their environment and the ability to fulfill their role within such environments as work, social activities, and relationships with partners and family"24,25. The current study revealed that patients with CSM with a preoperative SF score $<50$ have significantly greater improvement in their MCS score after laminoplasty than do patients with a preoperative SF score $>50$. This suggests that cervical myelopathy may have the greatest detrimental 
effect on social functioning among the numerous factors related to mental well-being (Figure 5). Since mental well-being is one of key factors for satisfaction with treatment ${ }^{12}$, it is crucial for physicians and patients to understand the influence of myelopathy, not only on the physical function of the upper and lower extremities, but also on social functioning. In addition, our results may suggest that adopting a different approach to this issue may lead to further improvements in the quality of life and patient satisfaction after surgery.

Several limitations of the present study need to be addressed. First, its retrospective nature rendered it difficult to definitively exclude not only inappropriate cases, but also potential biases, especially in regard to the referral of certain surgical procedures, the particular surgical techniques utilized, and a selection bias in the 5-year cohort. Second, although we analyzed the MCS score of the SF-36 as an indicator of mental well-being, mental health status should be analyzed in a multifaceted manner, and there may be other scores or questionnaires that are more suitable than the SF-36 MCS score as an assessment of psychological functioning. In addition, the value of the MCID can be affected by the calculation method, the anchor in anchor-based methods, and the patient cohort. Hence, the current results should be validated in further studies that take additional aspects into account. Finally, all of the patients were treated with laminoplasty. Other surgical methods, including posterior decompression and fusion, and anterior cervical discectomy and fusion, should be validated. However, the present study is the first to elucidate the 
predictors of an improvement in mental well-being after surgery and propound how myelopathy affects the patient's mental well-being.

\section{Conclusion}

The mental well-being of patients with CSM is impaired and, on average, improves after laminoplasty. The trend of improvement was sustained for 2 years, but not for 5 years. In terms of individual cases, half of the patients achieved an improvement that was greater than the MCID. A lower preoperative SF score, based on a cut-off value of 50, was an independent predictor of a significant improvement in the MCS score. The present results demonstrate the influence of CSM and subsequent surgical treatment on mental well-being. Recognizing this influence can aid patients with CSM and physicians in better understanding the potential for postoperative changes in mental well-being, and may help to develop other interventions for further improvement. 


\section{References}

1. Arrojas A, Jackson JB, 3rd, Grabowski G. Trends in the Treatment of Single and Multilevel Cervical Stenosis: A Review of the American Board of Orthopaedic Surgery Database. J Bone Joint Surg Am 2017;99:e99.

2. Hirabayashi K, Miyakawa J, Satomi K, et al. Operative results and postoperative progression of ossification among patients with ossification of cervical posterior longitudinal ligament. Spine (Phila Pa 1976) 1981;6:354-64.

3. Hirabayashi K, Toyama Y, Chiba K. Expansive laminoplasty for myelopathy in ossification of the longitudinal ligament. Clin Orthop Relat Res 1999:35-48.

4. Satomi K, Nishu Y, Kohno T, et al. Long-term follow-up studies of open-door expansive laminoplasty for cervical stenotic myelopathy. Spine (Phila Pa 1976) 1994;19:507-10.

5. Seichi A, Takeshita K, Ohishi I, et al. Long-term results of double-door laminoplasty for cervical stenotic myelopathy. Spine (Phila Pa 1976) 2001;26:479-87.

6. Wang MY, Green BA. Laminoplasty for the treatment of failed anterior cervical spine surgery. Neurosurg Focus 2003;15:E7.

7. Chow A, Mayer EK, Darzi AW, et al. Patient-reported outcome measures: the importance of patient satisfaction in surgery. Surgery 2009;146:435-43.

8. Godil SS, Parker SL, Zuckerman SL, et al. Determining the quality and effectiveness of surgical spine care: patient satisfaction is not a valid proxy. Spine J2013;13:1006-12.

9. Carr-Hill R, Sheldon T. Rationality and the use of formulae in the allocation of resources to health care. J Public Health Med 1992;14:117-26.

10. Tyser AR, Gaffney CJ, Zhang C, et al. The Association of Patient Satisfaction with Pain, Anxiety, and Self-Reported Physical Function. J Bone Joint Surg Am 2018;100:1811-8.

11. Abtahi AM, Brodke DS, Lawrence BD, et al. Association between patient-reported measures of psychological distress and patient satisfaction scores after spine surgery. J Bone Joint Surg Am 2015;97:824-8.

12. Kong CB, Jeon DW, Chang BS, et al. Outcome of spinal fusion for lumbar degenerative disease: a cross-sectional study in Korea. Spine (Phila Pa 1976) 2010;35:1489-94.

13. Hirabayashi K, Watanabe K, Wakano K, et al. Expansive open-door laminoplasty for cervical spinal stenotic myelopathy. Spine (Phila Pa 1976) 1983;8:693-9.

14. Kim KW, Lee DY, Jhoo JH, et al. Diagnostic accuracy of mini-mental status examination and revised hasegawa dementia scale for Alzheimer's disease. Dement Geriatr Cogn Disord 2005;19:324-30.

15. Tamai K, Suzuki A, Terai H, et al. Laminar closure after expansive open-door laminoplasty: fixation methods and cervical alignments impact on the laminar closure and surgical outcomes. Spine 
J2016;16:1062-9.

16. Association. JO. [Scoring system for cervical myelopathy.]. $J$ Jpn Orthop Assoc 1994;68:490-530.

17. Carreon LY, Djurasovic M, Dimar JR, 2nd, et al. Can the anxiety domain of EQ-5D and mental health items from SF-36 help predict outcomes after surgery for lumbar degenerative disorders? J Neurosurg Spine 2016;25:352-6.

18. Fukuhara S, Bito S, Green J, et al. Translation, adaptation, and validation of the SF-36 Health Survey for use in Japan. J Clin Epidemiol 1998;51:1037-44.

19. Strazdins E, Nie YF, Ramli R, et al. Association Between Mental Health Status and Patient Satisfaction With the Functional Outcomes of Rhinoplasty. JAMA Facial Plast Surg 2018;20:284-91.

20. Brazier JE, Fukuhara S, Roberts J, et al. Estimating a preference-based index from the Japanese SF-36. J Clin Epidemiol 2009;62:1323-31.

21. Badhiwala JH, Witiw CD, Nassiri F, et al. Minimum Clinically Important Difference in SF-36 Scores for Use in Degenerative Cervical Myelopathy. Spine (Phila Pa 1976) 2018;43:E1260-E6. 22. Simundic AM. [Diagnostic accuracy]. Acta Med Croatica 2006;60 Suppl 1:93-111.

23. Kato S, Oshima Y, Matsubayashi Y, et al. Minimum clinically important difference in outcome scores among patients undergoing cervical laminoplasty. Eur Spine J 2019.

24. McHorney CA, Ware JE, Jr., Raczek AE. The MOS 36-Item Short-Form Health Survey (SF-36): II. Psychometric and clinical tests of validity in measuring physical and mental health constructs. Med Care 1993;31:247-63.

25. Fukuhara S, Ware JE, Jr., Kosinski M, et al. Psychometric and clinical tests of validity of the Japanese SF-36 Health Survey. J Clin Epidemiol 1998;51:1045-53. 
Table1 Changes of average score $(n=111)$

\begin{tabular}{|c|c|c|c|}
\hline & Preoperative & 2-year postoperative & $\mathrm{p}$-value \\
\hline \multicolumn{4}{|l|}{ cJOA score (points) } \\
\hline Finger motion & $2.1 \pm 1.0$ & $3.0 \pm 1.1$ & $<0.001^{\#}$ \\
\hline U/E motion & $-0.4 \pm 0.5$ & $-0.1 \pm 0.3$ & $<0.001^{\#}$ \\
\hline L/E motion & $1.8 \pm 1.1$ & $2.5 \pm 1.1$ & $<0.001^{\#}$ \\
\hline U/E sensory & $0.9 \pm 0.5$ & $1.4 \pm 0.4$ & $<0.001^{\#}$ \\
\hline Trunk sensory & $1.7 \pm 0.5$ & $1.9 \pm 0.2$ & $<0.001^{\#}$ \\
\hline L/E sensory & $1.3 \pm 0.6$ & $1.7 \pm 0.3$ & $<0.001^{\#}$ \\
\hline BBD & $2.2 \pm 1.0$ & $2.6 \pm 0.7$ & $<0.001^{\#}$ \\
\hline Total point & $9.8 \pm 3.2$ & $13.3 \pm 2.5$ & $<0.001^{\#}$ \\
\hline \multicolumn{4}{|l|}{ VAS (mm) } \\
\hline neck pain & $24.2 \pm 27.8$ & $12.1 \pm 20.7$ & $<0.001^{\#}$ \\
\hline arm pain & $35.4 \pm 32.8$ & $17.2 \pm 26.8$ & $<0.001^{\#}$ \\
\hline arm numbness & $55.5 \pm 29.8$ & $35.7 \pm 29.9$ & $<0.001^{\#}$ \\
\hline \multicolumn{4}{|l|}{ SF-36 } \\
\hline $\mathrm{PF}$ & $42.3 \pm 30.4$ & $58.7 \pm 29.6$ & $<0.001^{\#}$ \\
\hline $\mathrm{RP}$ & $35.0 \pm 31.0$ & $57.8 \pm 32.1$ & $<0.001^{\#}$ \\
\hline BP & $36.5 \pm 23.7$ & $53.9 \pm 24.7$ & $<0.001^{\#}$ \\
\hline $\mathrm{GH}$ & $45.8 \pm 18.5$ & $49.4 \pm 18.8$ & 0.023 \\
\hline VT & $36.6 \pm 23.4$ & $51.6 \pm 23.5$ & $<0.001^{\#}$ \\
\hline $\mathrm{SF}$ & $52.7 \pm 31.7$ & $67.5 \pm 27.0$ & $<0.001^{\#}$ \\
\hline $\mathrm{RE}$ & $44.1 \pm 33.3$ & $61.9 \pm 33.0$ & $<0.001^{\#}$ \\
\hline MH & $52.6 \pm 25.3$ & $63.3 \pm 22.8$ & $<0.001^{\#}$ \\
\hline PCS & $24.0 \pm 13.5$ & $33.6 \pm 14.2$ & $<0.001^{\#}$ \\
\hline MCS & $40.7 \pm 13.4$ & $46.3 \pm 12.2$ & $<0.001^{\#}$ \\
\hline
\end{tabular}

\#: Paired-t test

cJOA: cervical Japanese Orthopaedic Association. VAS: visual analog scale, SF: Short form, PF: physical functioning, RP: physical role limitations, BP: bodily pain, GH: general health perceptions, VT: energy/vitality, SF: social functioning, RE: emotional role limitations, MH: mental health, PCS: physical component summary, MCS: mental component summary 
Table2 Preoperative factor relating with MCS improvement by more than the MCID

\begin{tabular}{|c|c|c|c|c|c|}
\hline & \multicolumn{3}{|c|}{ Univariate analysis } & \multicolumn{2}{|c|}{ Multivariate analysis } \\
\hline & $\begin{array}{l}\text { No-change } \\
\text { group } \\
(\mathrm{n}=55)\end{array}$ & $\begin{array}{l}\text { Improvement } \\
\text { group } \\
(\mathrm{n}=56)\end{array}$ & p-value & $\mathrm{aOR}$ & p-value \\
\hline Age & $66.0 \pm 11.1$ & $64.6 \pm 12.3$ & $0.557^{\#}$ & & \\
\hline Gender (female) & 33 & 32 & $0.848^{*}$ & & \\
\hline \multicolumn{6}{|l|}{ Preop cJOA score } \\
\hline Total score & $9.7 \pm 3.2$ & $9.9 \pm 3.2$ & $0.775^{\#}$ & & \\
\hline Finger motion & $2.0 \pm 0.9$ & $2.2 \pm 1.0$ & $0.257^{\#}$ & & \\
\hline U/E motion & $-0.4 \pm 0.5$ & $-0.4 \pm 0.5$ & 0.853 \# & & \\
\hline L/E motion & $1.9 \pm 1.0$ & $1.8 \pm 1.2$ & 0.540 \# & & \\
\hline U/E sensory & $0.9 \pm 0.4$ & $0.9 \pm 0.5$ & $0.490^{\#}$ & & \\
\hline Trunk sensory & $1.7 \pm 0.5$ & $1.7 \pm 0.5$ & $0.853^{\#}$ & & \\
\hline L/E sensory & $1.3 \pm 0.5$ & $1.3 \pm 0.6$ & $0.792^{\#}$ & & \\
\hline BBD & $2.3 \pm 1.0$ & $2.2 \pm 1.0$ & $0.605^{\#}$ & & \\
\hline \multicolumn{6}{|l|}{ Preop SF-36 } \\
\hline $\mathrm{PF}$ & $43.2 \pm 28.6$ & $41.4 \pm 32.0$ & $0.748^{\#}$ & & \\
\hline $\mathrm{RP}$ & $39.2 \pm 30.3$ & $30.9 \pm 31.1$ & $0.161^{\#}$ & & \\
\hline $\mathrm{BP}$ & $40.7 \pm 22.5$ & $32.3 \pm 24.0$ & $0.065^{\#}$ & & \\
\hline $\mathrm{GH}$ & $47.4 \pm 16.0$ & $44.3 \pm 20.6$ & $0.383^{\#}$ & & \\
\hline VT & $42.4 \pm 24.1$ & $30.9 \pm 21.2$ & $0.010^{\#}$ & 1.03 & 0.069 \\
\hline $\mathrm{SF}$ & $66.1 \pm 28.2$ & $39.5 \pm 29.4$ & $<0.001^{\#}$ & 0.97 & 0.001 \\
\hline $\mathrm{RE}$ & $55.6 \pm 32.4$ & $32.7 \pm 30.1$ & $<0.001^{\#}$ & 0.98 & 0.071 \\
\hline MH & $61.1 \pm 21.7$ & $44.2 \pm 25.7$ & $<0.001^{\#}$ & 0.98 & 0.076 \\
\hline
\end{tabular}

${ }^{\#}$ Mann-Whitney U test, ${ }^{*}$ Chi-squared test. Preop: Preoperative, cJOA: cervical Japanese Orthopaedic Association, U/E:

upper extremity, L/E: lower extremity, BBD: bowel bladder disfunction, SF: Short form, PF: physical functioning, RP:

physical role limitations, BP: bodily pain, GH: general health perceptions, VT: energy/vitality, SF: social functioning,

RE: emotional role limitations, MH: mental health 
Table3 Change of each score after surgery

\begin{tabular}{lccc}
\hline & $\begin{array}{c}\text { No-change group } \\
(\mathrm{n}=55)\end{array}$ & $\begin{array}{c}\text { Improvement group } \\
(\mathrm{n}=56)\end{array}$ & p-value \\
\hline Change of cJOA score & $3.3 \pm 2.9$ & $3.6 \pm 2.9$ & $0.551^{\#}$ \\
Change of SF-36 score & & & \\
PF & $11.0 \pm 24.9$ & $21.7 \pm 29.1$ & $0.041^{\#}$ \\
RP & $14.3 \pm 26.8$ & $31.0 \pm 35.0$ & $0.006^{\#}$ \\
BP & $7.7 \pm 21.0$ & $27.1 \pm 27.2$ & $<0.001^{\#}$ \\
GH & $-0.8 \pm 14.1$ & $17.4 \pm 7.9$ & $0.005^{\#}$ \\
VT & $4.2 \pm 16.8$ & $25.6 \pm 20.4$ & $<0.001^{\#}$ \\
SF & $-7.0 \pm 28.8$ & $36.2 \pm 30.6$ & $<0.001^{\#}$ \\
RE & $-0.9 \pm 29.7$ & $36.3 \pm 33.1$ & $<0.001^{\#}$ \\
MH & $-2.4 \pm 14.2$ & $23.5 \pm 21.5$ & $<0.001^{\#}$ \\
\hline
\end{tabular}

\# Mann-Whitney U test, ${ }^{*}$ Chi-squared test. Preop: Preoperative, cJOA: cervical Japanese Orthopaedic Association, U/E:

upper extremity, L/E: lower extremity, BBD: bowel bladder disfunction, SF: Short form, PF: physical functioning, RP:

physical role limitations, BP: bodily pain, GH: general health perceptions, VT: energy/vitality, SF: social functioning,

RE: emotional role limitations, MH: mental health 


\section{Figure legends}

Figure1 Trend of MCS score improvement during 2-year postoperatively (n=111)

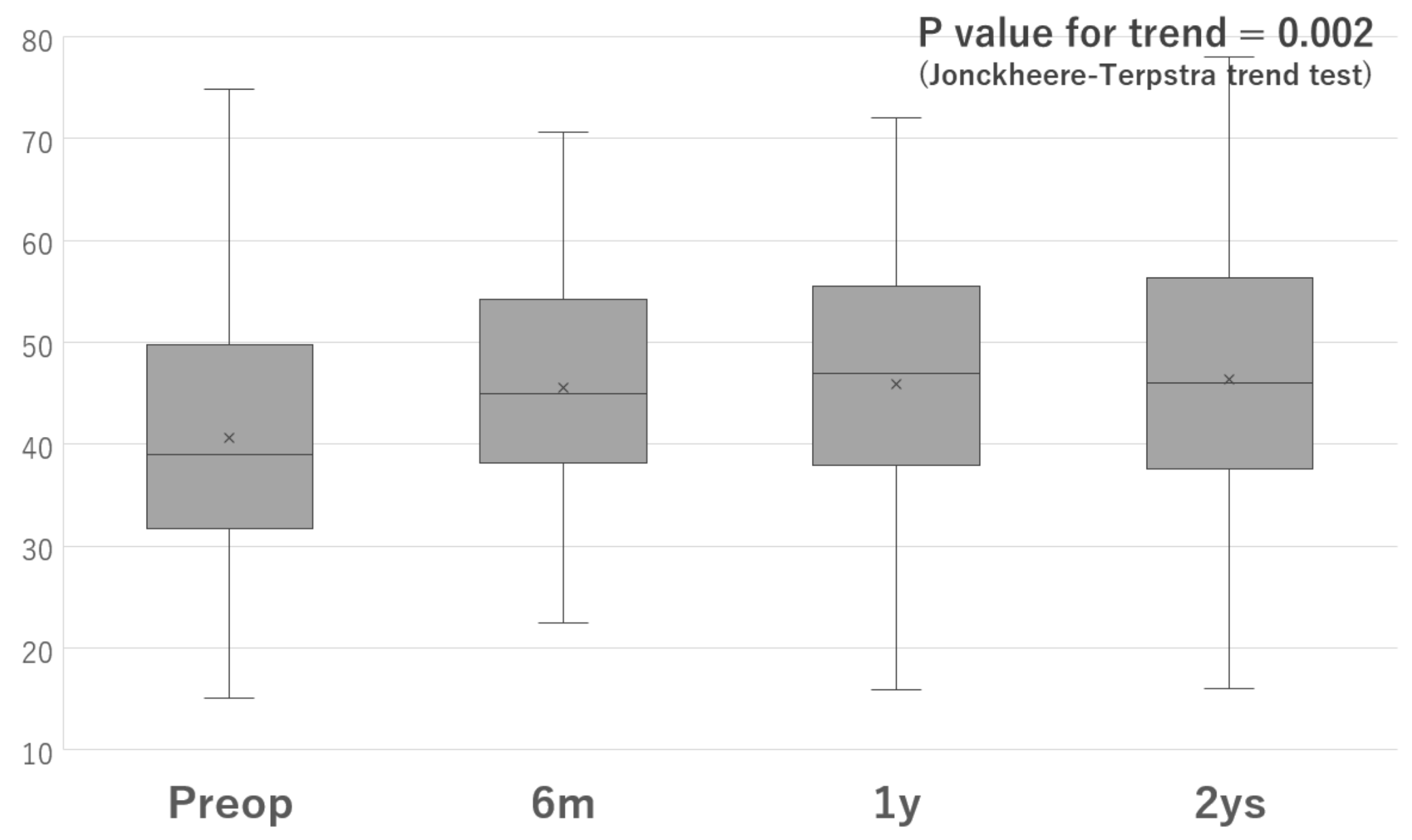


Figure2 ROC curve analysis of SF to predict the MCS score improvement $>$ MCID at 2-year ( $n=111$ )

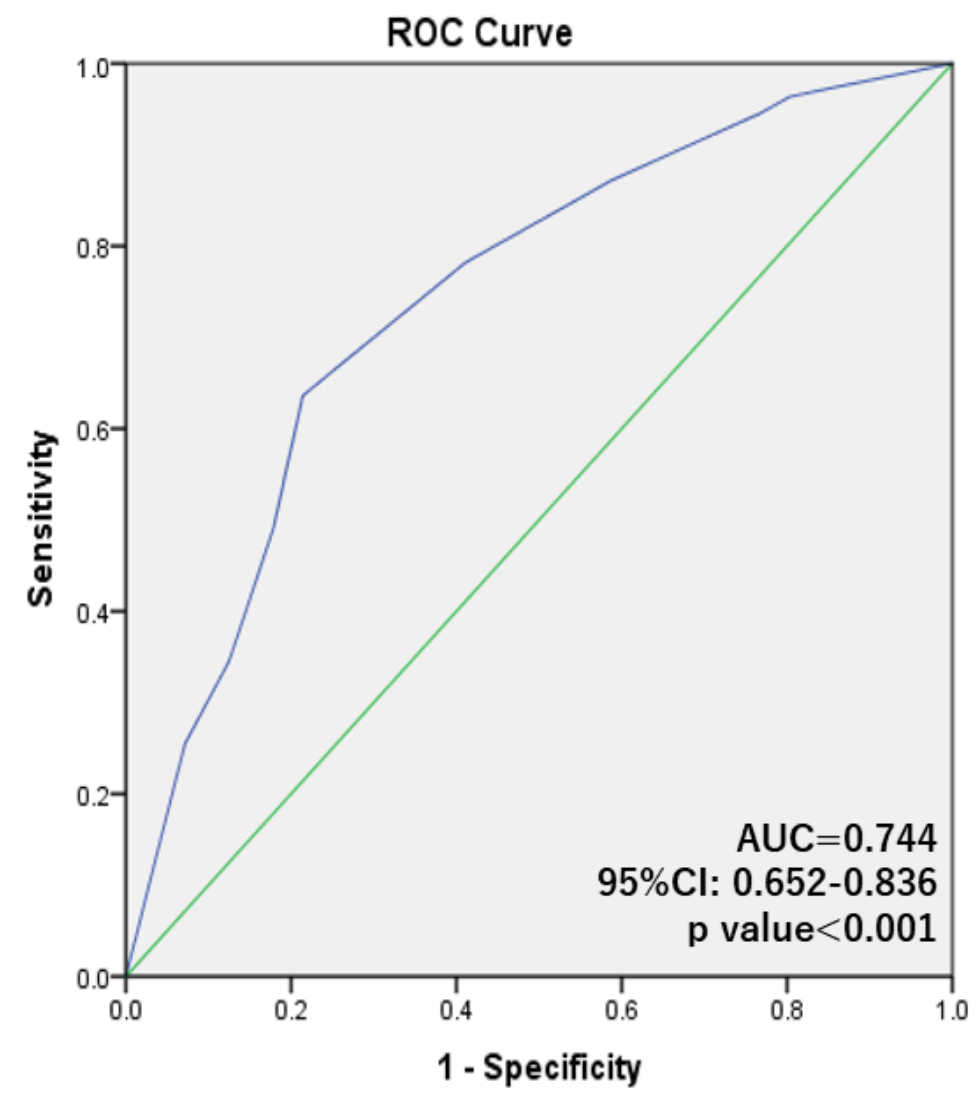

Diagonal segments are produced by ties. 
Figure 3 Change of MCS score at 5-year postoperatively $(n=43)$

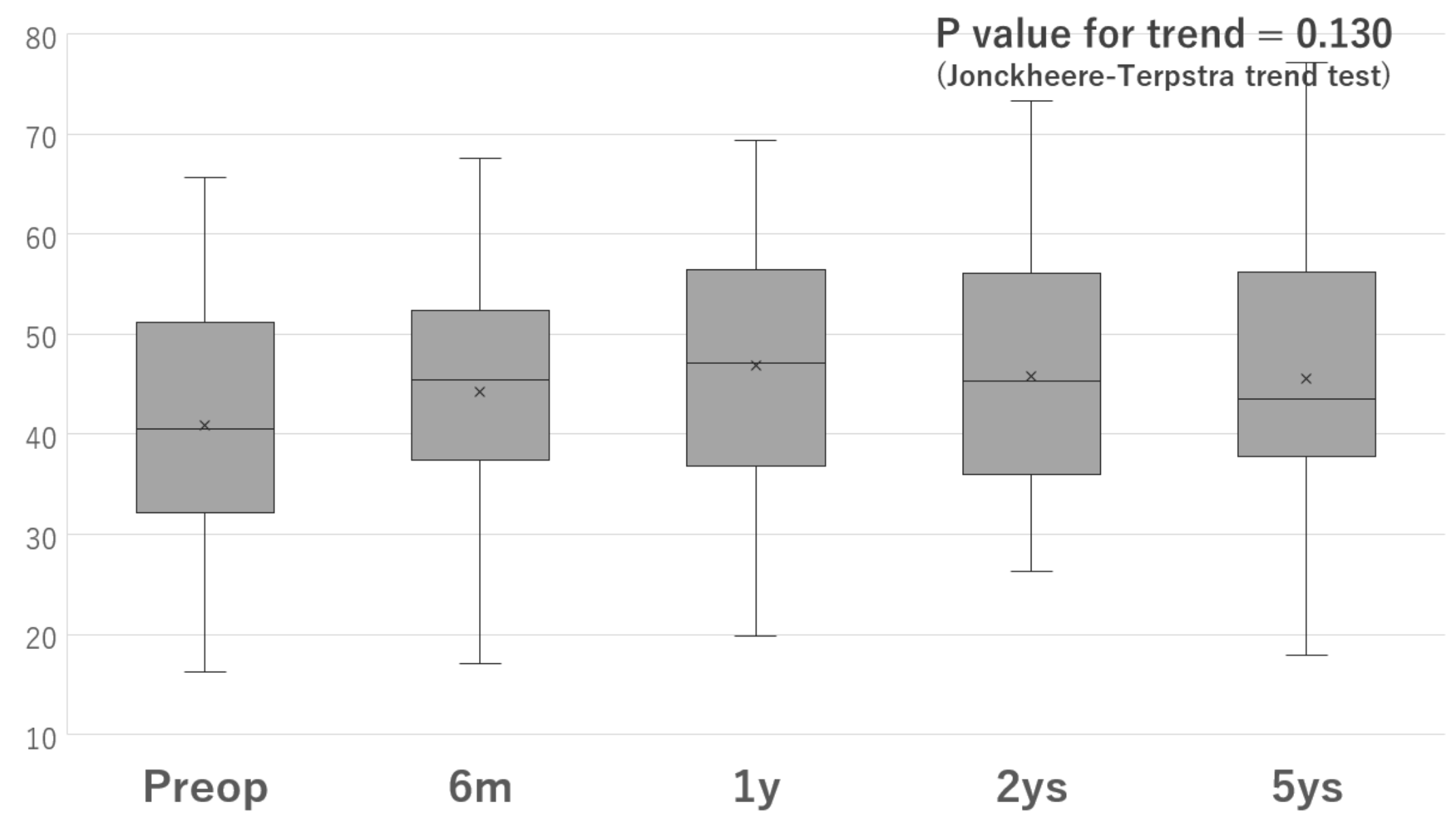


Figure 4 ROC curve analysis of SF to predict the MCS score improvement $>$ MCID at 5-year ( $n=43$ )

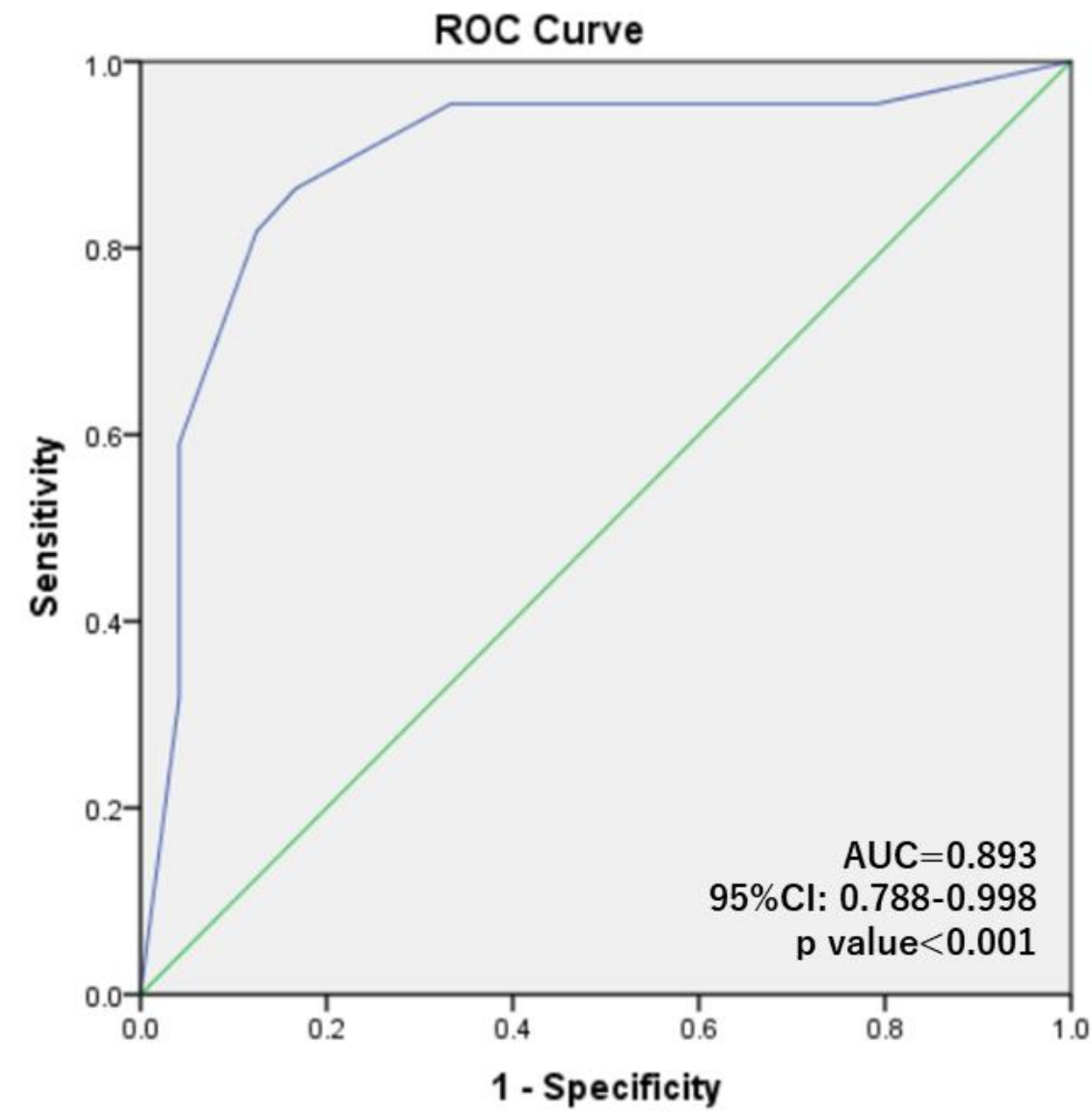




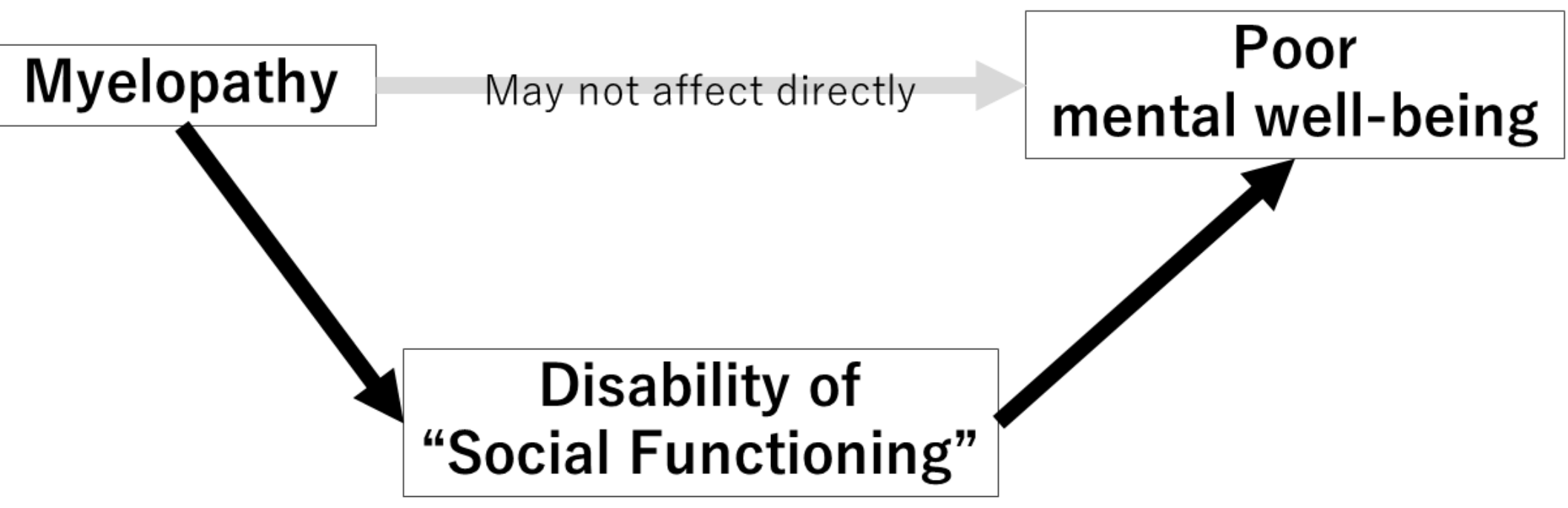

